\title{
SUSCEPTIBILITY TO EXPERIMENTAL PYELONEPHRITIS WHEN HORMONAL HYPERTENSION IS PREVENTED BY HYPOTENSIVE DRUGS *
}

\author{
By JAMES W. WOODS \\ (From the Department of Medicine, University of North Carolina School of Medicine, \\ Chapel Hill, N. C.)
}

(Submitted for publication April 29, 1960; accepted August 3, 1960)

Rats with desoxycorticosterone (DCA)-saline induced hypertension have been shown to exhibit increased susceptibility to experimental pyelonephritis $(1,2)$. These studies lend support to the hypothesis that various injuries, including nephrosclerosis, predispose to human pyelonephritis and that pyelonephritis is superimposed upon hypertensive disease more frequently than is usually suspected. Others (3-5) have shown that reserpine and/or hydralazine, when given to rats with DCA-saline hypertension, will reduce blood pressure, delay or prevent renal and vascular pathological changes, and increase the average survival time. Masson, McCormack, Dustan and Corcoran (6) demonstrated a close association between blood pressure levels and the presence and character of the vascular lesions in rats with hydralazine-treated renal hypertension. The previously demonstrated susceptibility of rats with DCA-saline hypertension might have been due either to an effect of the hypertension and vascular disease or to some other effect of the administered steroid and saline. The present investigation was therefore designed to separate these possibilities.

\section{MATERIALS AND METHODS}

White, female Sprague-Dawley strain rats, weighing 100 to $150 \mathrm{~g}$, were used. The experimental animals of Groups A and B were subjected to left nephrectomy and 3 fortnightly injections of a long-acting derivative of DCA 1 in $25 \mathrm{mg}$ doses. Drinking water contained 1 per cent $\mathrm{NaCl}$ and 0.49 per cent $\mathrm{KCl}$. The latter had been previously found (1) to prevent potassium depletion which otherwise results from the DCA-saline regimen. In addition, $40 \mathrm{mg}$ of hydralazine 1 was added to each liter of the drinking solution of Group $A$ animals and they received $1 \mathrm{mg}$ per $\mathrm{kg}$ (later reduced to $0.5 \mathrm{mg}$ per

* This investigation was supported by a grant from The Life Insurance Medical Research Fund.

1 Kindly supplied by Ciba Pharmaceutical Products, Inc., Summit, N. J. $\mathrm{kg}$ ) of syrosingopine ${ }^{1}$ subcutaneously each day. Syrosingopine was chosen rather than reserpine since it has been found to have little, if any, effect on spontaneous salt appetite (7).

The animals of Group C had a left nephrectomy but received no DCA and drank ordinary tap water. All animals ate Purina Laboratory Chow. The animals were "pair-fed" by groups, i.e., the intakes of Groups A and C were limited each day to the average intake of Group B. Additionally, the fluid intake of Group B animals was limited each day to the average fluid intake of Group A in order to adjust for any effect syrosingopine might have on spontaneous salt intake. The resulting weight curves of the three groups were very similar, as is shown in Figure 1.

The method of determining blood pressure by use of a microphonic manometer and the bacteriological techniques employed have been previously described $(1,8)$. Rats of the three groups designated for bacterial injection received an inoculum of $100,000,000$ Escherichia coli i.v.

Experimental design. The experimental design is shown in Table I. Administration of hypotensive drugs and the DCA-saline regimen were started simultaneously.

Intravenous injection of $E$. coli was carried out 7 weeks later and the rats sacrificed 2 weeks following injection. Six rats of Groups $A$ and $B$ and 5 of Group $C$ did not receive $E$. coli, in order to facilitate histological study of

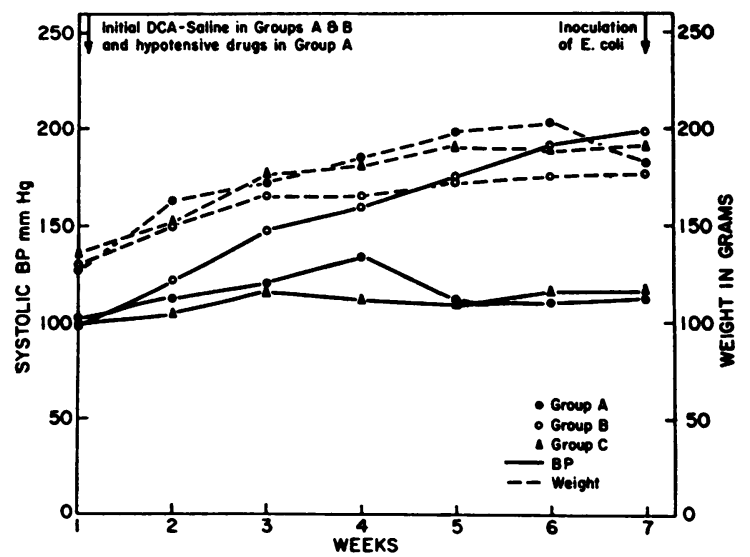

Fig. 1. Average weights and Systolic blood Pressures of Groups A, B AND C. 


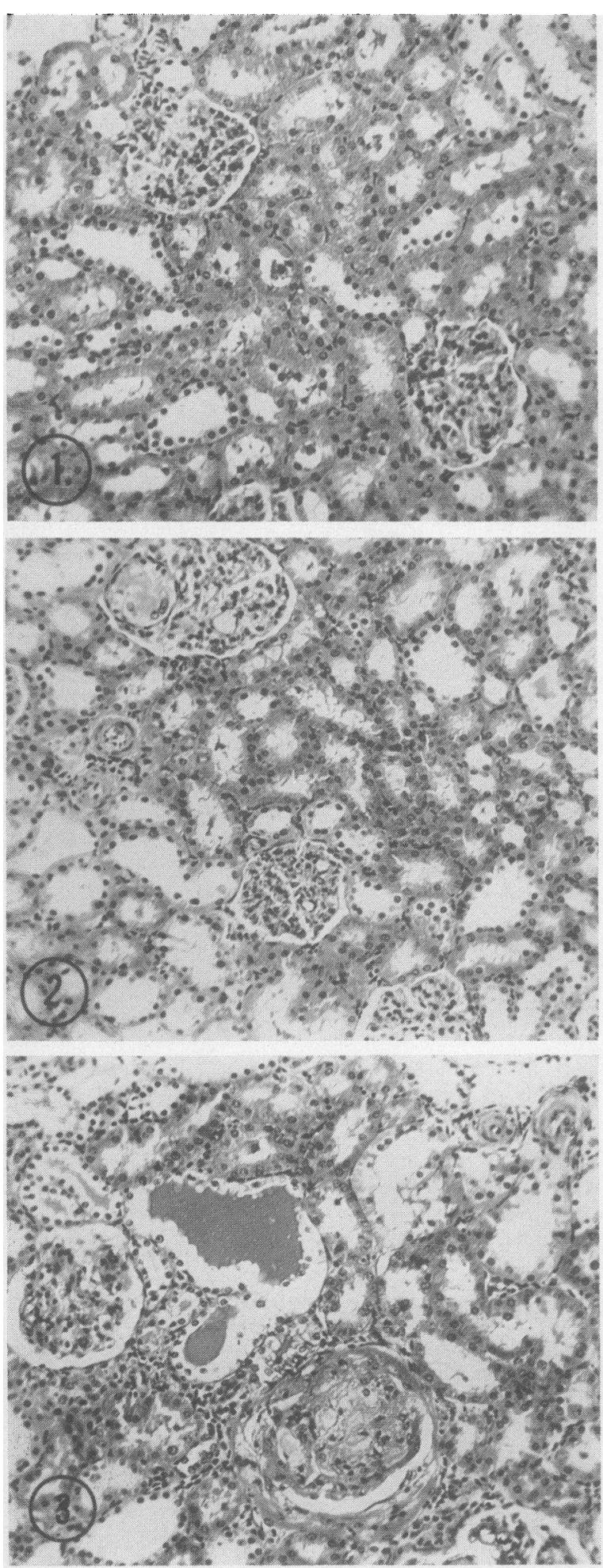

Fig. 2. Illustrations of NORMAL AND Pathological HISTOLOGY ENCOUNTERED. 1. Kidney of control rat (previously uninephrectomized). 2. Kidney from an uninjected rat of Group A showing a nodular deposit of material
TABLE I

Experimental design

\begin{tabular}{cccc} 
& \multicolumn{1}{c}{ Description } & $\begin{array}{c}\text { No. of rats } \\
\text { receiving } \\
\text { E. coli i.v. }\end{array}$ & $\begin{array}{c}\text { No. of rats } \\
\text { uninjected } \\
\text { with } E \text {. coli }\end{array}$ \\
\hline $\mathrm{A}$ & $\begin{array}{l}\text { DCA-saline regimen } \\
\text { uninephrectomy } \\
\text { syrosingopine-hydralazine }\end{array}$ & 15 & 6 \\
$\mathrm{~B}$ & $\begin{array}{l}\text { DCA-saline regimen } \\
\text { uninephrectomy } \\
\text { no hypotensive drugs }\end{array}$ & 16 & 6 \\
$\mathrm{C} \quad$ Ininephrectomized controls & 14 & 5 \\
\hline
\end{tabular}

the kidneys of these representatives after sacrifice. At the time of sacrifice, the remaining right kidney of each animal was removed with aseptic technique, divided in half longitudinally, and one-half cultured quantitatively while the other half was fixed in 10 per cent formalin for histological sections.

\section{RESULTS}

General condition of animals. While equal numbers (nine) of rats died in Groups $\mathrm{A}$ and $\mathrm{B}$ prior to injection of $E$. coli there appeared to be a difference between the two groups. All of the Group A rats were markedly edematous at the time of death and six of nine died within the first eight days of the experiment. This, correctly or not, was attributed to the syrosingopine, and the mortality rate and development of edema seemed to decline markedly when the dose of this drug was reduced from 1 to $0.5 \mathrm{mg}$ per $\mathrm{kg}$ per day. Deaths of the nine rats of Group B were fairly evenly distributed over the interval of 6 to 20 days after onset of the experiment and all but one were thin and sickly in appearance. The one exception was edematous. Four of the control animals in Group $\mathrm{C}$ also died-one accidentally, one from intestinal obstruction, and two from unknown cause. The remaining animals of the three groups maintained body weight, appeared reasonably healthy, and all survived to the time of sacrifice, two weeks following intravenous injection of $E$. coli.

Systolic blood pressure. As is seen in Figure 1, the combination of syrosingopine and hydralazine was highly successful in preventing severe hyper-

with the staining characteristics of fibrinoid in a glomerulus and very slight tubular dilatation. 3. Kidney from an uninjected rat of Group B showing periglomerular fibrosis, glomerular sclerosis, marked dilatation of tubules, some of which contain casts, and minimal arteriolar sclerosis. Hematoxylin and eosin $(\times 200)$. 
tension. The average of the blood pressures of the Group A rats was practically identical with that of the controls of Group $\mathrm{C}$ at the time of bacterial injection. There were individual variations in blood pressure levels, but no animal in this group had a reading above $160 \mathrm{~mm} \mathrm{Hg}$ and most had pressures below $130 \mathrm{~mm}$ during the greater part of the seven week period. The average blood pressure for Group B, on the other hand, rose progressively to $200 \mathrm{~mm} \mathrm{Hg}$ by the end of seven weeks. The highest pressure recorded in this group was $236 \mathrm{~mm} \mathrm{Hg}$.

Renal histology. The rapid development of hypertension in the albino rat as a result of the DCA-saline regimen is usually accompanied by the appearance of widespread acute vascular disease $(5,9)$. Arteriolar sclerosis is present and some arterioles contain subendothelial material with the characteristics of fibrinoid. Glomeruli show enlargement and capsular fibrosis. Convoluted tubules are often dilated and contain eosinophilic colloid. The above described lesions were present to a moderate or severe degree in the kidneys of rats of Group B. In most of the rats of Group A, there were no or minimal glomerular and tubular changes, and fibrinoid arteriolar lesions were rare. The two instances in this group of more advanced renal changes also had the highest systolic blood pressure recorded. Illustrations of kidneys of normal (uninephrectomized) rats, and of those of Groups A and B are shown in Figure 2.
Susceptibility to experimental pyelonephritis. The results are shown in Table II. Of the 15 rats of Group A receiving one hundred million E. coli intravenously, 4 had unequivocal pyelonephritis. One had no histological evidence of infection, a colony count of only 800 bacteria per $\mathrm{ml}$ of renal tissue, and was considered uninfected. Of the 16 rats of Group B, 10 had clear-cut evidence of pyelonephritis. One animal had both gross and microscopic evidence of abscess formation in the half-kidney which was fixed for sections, while the remaining half had no gross abscesses and was sterile on culture. The null-hypothesis stating that the incidence of infection is the same for rats in Groups A and $B$ was rejected in favor of the alternative hypothesis that if a difference did in fact exist, it would be in the direction of rats in Group B having a greater incidence of infection than rats in Group A. This conclusion was arrived at after application of the appropriate one-tailed test of significance based upon Fisher's exact method for a $2 \times 2$ contingency table $(10)$. The calculated probability of an error of the first kind (viz., that of falsely concluding that the incidence of infection for rats in Group $B$ is greater than that for rats in Group A) is $p=0.049$. Only one control of Group $C$ had renal infection. This was evidenced by a microscopic abscess and a colony count of 9,500 organisms per $\mathrm{ml}$ of kidney. The difference in incidence of infection between Groups $B$ and $C$ is highly significant $(p=0.002)$, as was

TABLE II

Colony counts and descriptions of kidneys of the three groups two weeks after injection of E. coli

\begin{tabular}{|c|c|c|c|c|c|}
\hline \multicolumn{2}{|c|}{ Group A } & \multicolumn{2}{|c|}{ Group B } & \multicolumn{2}{|c|}{ Group C } \\
\hline Colony counts & Description* & Colony counts & Description & Colony counts & Description \\
\hline 0 & 0 & $1,200,000$ & ++ & 0 & 0 \\
\hline $3,700,000$ & $+t+$ & $8,000,000$ & $++t$ & 0 & 0 \\
\hline 800 & 0 & $11,000,000$ & $++t$ & 0 & 0 \\
\hline $5,000,000$ & $+t+$ & $2,000,000$ & $+t+$ & 0 & 0 \\
\hline $20,000,000$ & $++t$ & $2,100,000$ & ++ & 0 & 0 \\
\hline $2,000,000$ & ++ & 0 & $+t+$ & 0 & 0 \\
\hline 0 & 0 & 100,000 & ++ & 0 & 0 \\
\hline 0 & 0 & $1,400,000$ & $++t$ & 0 & 0 \\
\hline 0 & 0 & $18,000,000$ & ++ & 0 & 0 \\
\hline 0 & 0 & 0 & 0 & 0 & 0 \\
\hline 0 & 0 & 0 & 0 & 9,500 & $+t$ \\
\hline 0 & 0 & $100,000,000$ & $++t$ & 0 & 0 \\
\hline 0 & 0 & 0 & 0 & 0 & 0 \\
\hline 0 & 0 & 0 & 0 & 0 & 0 \\
\hline \multirow[t]{2}{*}{0} & 0 & 0 & 0 & & \\
\hline & & 0 & 0 & & \\
\hline
\end{tabular}

$* 0$, no cellular infiltrate;,++ microscopic abscesses;,+++ macroscopic abscesses. 
previously found (1). There was no significant difference in incidence of infection between Groups $\mathrm{A}$ and $\mathrm{C}$.

\section{DISCUSSION}

The renewal of interest in and great increase in research on pyelonephritis and unilateral renal disease associated with hypertension in recent years have raised many questions regarding the interrelationships of these diseases in man. Few would question that chronic bilateral pyelonephritis may produce severe hypertensive disease, but it often does not, even when leading to uremia. Much skepticism has arisen regarding the role of unilateral pyelonephritis and hypertension, since removal of the affected kidney so rarely results in alleviation of the hypertension. Even the production of hypertension in the experimental animal by means of pyelonephritis has not been firmly established. If such an experimental model can be developed, it should be quite helpful in the elucidation of these problems.

The reverse relationship, i.e., that hypertensive renal disease may predispose to pyelonephritis, is only suggested so far by a few human and animal investigations $(1,2,11,12)$. Goldblatt (13) has hypothesized that malignant hypertension may develop from benign hypertension because of the superimposition of renal infection. Long-term studies of human normal and hypertensive populations will be required to settle these questions.

The data reported here again show that hypertensive disease induced by DCA and saline leads to greatly increased susceptibility to experimental pyelonephritis. They also suggest that the blood pressure itself is an important factor in the production of renal damage and heightened susceptibility, since when it is controlled at normal or near normal levels, renal arterial and arteriolar lesions are minimal or absent and susceptibility to infection is reduced. That the blood pressure may not be the only factor is demonstrated by the recent experiments of Gardner (5). He showed that, in rats with steroid hypertension receiving daily intramuscular injections of hydralazine, acute vascular disease can be prevented despite discontinuous reduction in the mean systemic blood pressure and suggested that this prevention was due either to the discontinuous effect on blood pressure or to "a factor, at present unrecognized, which is more sensitive to the action of the drug than is the blood pressure." In this study, hydralazine was present in the drinking solution, syrosingopine was administered additionally, and a continuous hypotensive effect is assumed. However, it cannot be proved, since blood pressure measurements were made at weekly intervals.

This study has made no attempt to investigate the effect of hypotensive drugs on survival time of the animals and, while the doses of syrosingopine used during the first week of the experiment in Group A seemed to cause edema and early death, this is not certain. In this investigation, there was no significant difference in mortality rate during the seven week period prior to bacterial injection between the rats with controlled and untreated hormonal hypertension.

\section{SUMMARY}

Rats with desoxycorticosterone-saline induced hypertension have again been found to exhibit marked renal vascular damage and increased susceptibility to experimental pyelonephritis. In other rats on the same regimen to which hypotensive drugs were added, blood pressure was at or near normal levels when measured at weekly intervals, renal vascular lesions were minimized, and no increased susceptibility to pyelonephritis was demonstrated.

\section{ACKNOWLEDGMENT}

The author appreciates the technical assistance of Miss Billie Sioux Bush and is indebted to Dr. Bernard Pasternack, Department of Biostatistics, School of Public Health, for help with the statistical analysis and to Dr. William Huffines, Department of Pathology, for help with analysis of histological sections.

\section{REFERENCES}

1. Woods, J. W. Susceptibility of rats with hormonal hypertension to experimental pyelonephritis. J. clin. Invest. 1958, 37, 1686.

2. Shapiro, A. P. Relationships of hypertension and renal impairment to experimental chronic pyelonephritis in rats (abstract). J. clin. Invest. 1958, 37, 930.

3. Gaunt, R., Antonchak, N., Miller, G. J., and Renzi, A. A. Effect of reserpine (Serpasil) and hydralazine (Apresoline) on experimental steroid hypertension. Amer. J. Physiol. 1955, 182, 63.

4. Gross, F., Noelpp, B., Sulser, F., Doebelin, R., and Kündig, H. Vergleichende Untersuchungen über die medikamentöse Beeinflussung verschiedener 
Formen von experimenteller Hypertension. Klin. Wschr. 1955, 33, 372.

5. Gardner, D. L. The relationship between intermittent hypotension and the prevention by hydralazine of acute vascular disease in rats with steroid hypertension. Brit. J. exp. Path. 1960, 41, 60.

6. Masson, G. M. C., McCormack, L. J., Dustan, H. P., and Corcoran, A. C. Hypertensive vascular disease as a consequence of increased arterial pressure. Quantitative study in rats with hydralazine-treated renal hypertension. Amer. J. Path. 1958, 34, 817.

7. Gaunt, R., Gross, F., Renzi, A. A., and Chart, J. J. Adrenal cortex in hypertension in Hypertension, J. H. Moyer, Ed. First Hahnemann Symp. on Hypertensive Disease. Philadelphia, Saunders, 1959, p. 225.

8. Woods, J. W., Welt, L. G., Hollander, W., Jr., and Newton, M. Susceptibility of rats to experimental pyelonephritis following recovery from potassium depletion. J. clin. Invest. 1960, 39, 28.

9. Selye, H., Hall, C. E., and Rowley, E. M. Malignant hypertension produced by treatment with desoxycorticosterone acetate and sodium chloride. Canad. med. Ass. J. 1943, 49, 88.

10. Fisher, R. A. Statistical Methods for Research Workers, 12th ed. Edinburgh, Oliver and Boyd, 1954.

11. Smythe, C. M., Rivers, C. F., and Rosemond, R. M. Incidence of infected urines in hypertensive subjects (abstract). Amer. J. Med. 1959, 27, 325.

12. Grieble, H. G., Johnston, L. C., and Jackson, G. G. A search for unsuspected pyelonephritis among patients with hypertension (abstract). Clin. Res. 1958, 6, 293.

13. Goldblatt, $\mathrm{H}$. Pathogenesis of malignant hypertension (editorial). Circulation 1957, 16, 697. 